\title{
Correction to: Infections in Childhood
}

\author{
Vivek Yedavalli, Vivek Pandey, and Delilah Burrowes
}

\section{Correction to: B. Gao et al. (eds.), Imaging of CNS Infections and Neuroimmunology, https://doi.org/10.1007/978-981-13-6904-9_13}

The author name in chapter 13 was misspelled as Vivek Yedavilli whereas the correct spelling should be Vivek Yedavalli. This has been corrected in all formats of the book. 